\title{
Estimation of the sugar content of fruit by energy-resolved computed tomography using a material decomposition method
}

\author{
$\operatorname{AUTHOR(S):~}$ \\ Kanno, Ikuo; Kuroyama, Takahiro
}

\section{CITATION:}

Kanno, Ikuo ... [et al]. Estimation of the sugar content of fruit by energy-resolved computed tomography using a material decomposition method. Journal of Nuclear Science and Technology 2021, 58(5): 533-541

\section{ISSUE DATE:}

2021

URL:

http://hdl.handle.net/2433/263000

\section{RIGHT:}

This is an Accepted Manuscript of an article published by Taylor \& Francis in Estimation of the sugar content of fruit by energy-resolved computed tomography using a material decomposition method on 2020/11/23, available online: https://www.tandfonline.com/10.1080/00223131.2020.1845836.; The full-text file will be made open to the public on 23 Nobember 2021 in accordance with publisher's 'Terms and Conditions for Self-Archiving'.: This is not the published version. Please cite only the published version.この論文は出版社版でありません。引用の際には出版社版をご確認じ 利用ください。 
1 Estimation of the sugar content of fruit by energy-resolved computed

2 tomography using a material decomposition method

3 Ikuo Kanno* and Takahiro Kuroyama

4 Department of Nuclear Engineering, Graduate School of Engineering, Kyoto University,

5 Katsura, Nishikyo, Kyoto 615-8530, Japan

6 *kanno@nucleng.kyoto-u.ac.jp

7 


\section{Estimation of the sugar content of fruit by energy-resolved computed 9 tomography using a material decomposition method}

We have been developing energy-resolved X-ray computed tomography (ER-CT) using a novel detector system called a transXend detector, which measures Xrays as electric current and gives the energy distribution of X-rays by unfolding analysis. This paper presents the results of the sugar content measurements of kiwifruit samples by ER-CT. Because the change of linear attenuation coefficients as a function of sugar content was small, we used a material decomposition method instead of the unfolding method to deduce the linear attenuation coefficient from the measured electric current. The obtained sugar contents showed good agreement with those measured by a refractometer.

Keywords: X-ray; computed tomography; energy distribution; material decomposition; non-destructive; experiment

\section{Introduction}

X-ray computed tomography $(\mathrm{CT})$ is an effective modality to identify tumor tissue and anomalies inside the organs of human bodies. In CT inspections at hospitals, X-rays are measured as electric current without using the energy information of X-ray photons. This measurement method is called a current measurement. X-rays are attenuated according to the linear attenuation coefficient of a material. The linear attenuation coefficient is generally greater for X-rays of lower energy according to data from the National Institute of Standards and Technology (NIST) [1]. As X-rays pass through a material, the number of low-energy X-ray photons decreases more quickly than that of high-energy X-ray photons, causing the number of high-energy X-ray photons to dominate. As a result, the averaged energy of an X-ray energy spectrum increases after passing through a material. The measured linear attenuation coefficient of the material 
34 changes as a function of the path length of the X-rays. This phenomenon is called the 35 beam hardening effect [2].

To avoid the beam hardening effect, it is necessary to use the energy information

of X-rays [3]. There are three ways to exploit the energy information of X-rays in CT:

dual-energy CT (DE-CT), photon-counting CT (PC-CT) and energy-resolved CT (ER-

39 CT).

DE-CT performs CT measurements twice with changing X-ray tube voltages [4].

41 The change of averaged X-ray energies is, however, not much. When using $80 \mathrm{kV}$ and $140 \mathrm{kV}$ tube voltages, typical averaged energies are $42.4 \mathrm{keV}$ and $58.2 \mathrm{keV}$, respectively. DE-CT gives not enough energy information. Moreover, DE-CT results in

44 large exposure dose due to two exposures. As an alternative of DE-CT, dual-layer detector is used to give linear attenuation coefficients for high and low energy X-rays [5]. Again, the difference of averaged energies measured by the upper and lower detector is not much.

In PC-CT, the energy of each incident X-ray photon is measured and sorted into 6 to 8 energy bins. The number of X-rays coming into a CT detector, however, is too high to perform the energy measurement of each X-ray photon. With this difficulty, the photon counting CT is under study with suppressed counting rate [6]. The systems of PC-CT are in the pre-clinical investigation stage [7]. To compete with DE-CT, further improvement is necessary [8].

We have been studying energy-resolved X-ray computed tomography (ER-CT) using a novel detector system called a "transXend" detector [9]. The transXend detector consists of multiple segmented detectors aligned in the direction of X-ray

57 incidence as shown in Figure 1 of Ref. [9]. We call this a stacked-type transXend 58 detector. Because each segmented detector measures X-rays as electric current, the 
transXend detector has no problems associated with the huge number of incident X-rays.

60 Using the previously obtained response functions for each segmented detector as a

61 function of X-ray energy, the energy distribution of incident X-rays can be estimated by

62 an unfolding method for the X-ray energy with $0.5 \mathrm{keV}$ or $1 \mathrm{keV}$ interval. We

63 measured the linear attenuation coefficients of nine kinds of resins and estimated their

64 effective atomic numbers $\left(Z_{\text {eff }}\right)$, which ranged from 5 to 14 with the errors of $Z_{\text {eff }}$ were

65 within $3 \%[10]$.

Apart from the stacked-type transXend detector for the measurement with pencil

beam X-rays, we developed a two-dimensional transXend detector with a flat panel detector (FPD) and two kinds of ribbon metal absorbers in lattice structure in front of

69 the FPD [11]. For CT measurements with the stacked-type transXend detector, a

70 phantom was scanned by pencil beam X-rays with the movements of translation and rotation. This measurement takes long time. With the two-dimensional transXend detector, the phantom is scanned by fan beam or cone beam X-rays with the rotation movement. The two-dimensional transXend detector has an advantage of shorter measurement time, which advantage is the same one for the third generation CT to the first generation CT.

We measured $Z_{\text {eff }}$ of eleven RMI rods (Gammex, Middleton, WI, US) which were developed by Constantinou, et al. [12] with ER-CT method. $Z_{\mathrm{eff}}$ of rod materials were measured within $\pm 1.8 \%$ error after the normalization with the one of breast [13].

79 Similar measurements were performed by some groups with using the RMI rods by

80 clinical CT scanners with DE-CT method. Bazalova et al. performed DE-CT with 100

$81 \mathrm{kV}$ and $140 \mathrm{kV}$ tube voltages and reported $Z_{\text {eff }}$ with averaged error $2.8 \%$, with the largest error $12.0 \%$ [14]. Goodsitt et al. used rapid $\mathrm{kVp}$ switching DE-CT method and 
83 gave errors of $-6 \%$ to $+6 \%$ for phantoms with $Z_{\text {eff }}>6.3$, and the errors of $15 \%$ for 84 phantoms with $Z_{\text {eff }}<6.3$, respectively [15].

In this paper, we describe a method to use ER-CT to estimate the sugar content of fruit as a precise measurement of $Z_{\text {eff. }}$ The $Z_{\text {eff }} \mathrm{S}$ of fructose and water are 6.95 and 7.42, respectively. Similar to the $Z_{\text {eff }}$ estimation of resins, sugar content is determined by measuring the linear attenuation coefficient of a sample. However, the change of the linear attenuation coefficient as a function of sugar content is small. To distinguish small differences of linear attenuation coefficients, we employ a material decomposition method to analyze the ER-CT data instead of unfolding the X-ray energy distribution, which we used previously.

Generally, sugar content is measured in the unit of degrees Brix $\left({ }^{\circ} \mathrm{Bx}\right)$; the sugar content of a $100-\mathrm{g}$ solution containing $1 \mathrm{~g}$ of sucrose at $20^{\circ} \mathrm{C}$ is defined $1{ }^{\circ} \mathrm{Bx}$. Sucrose consists of glucose and fructose, which are isomers of each other.

Sugar content measurement methods include destructive and non-destructive approaches. A sugar content meter that measures the refractive index of liquids, i.e., the juice of a fruit, is a destructive method and has an accuracy of $0.1 \%-0.2 \%$ [16].

99 Infrared (IR) spectroscopy is a non-destructive method to estimate the sugar content of 100 fruit that involves with measuring the absorption spectrum of IR light and estimating 101 sugar content using the previously obtained calibration line of IR light absorption.

102 However, IR spectroscopy has some disadvantages, including measurement error 103 caused by the variation of fruit samples from those used to make the calibration line and 104 restricted measurement region that is limited to the fruit surface where IR light can reach. A typical commercial IR Brix meter has an accuracy of 1.0\%-1.5\% [16]. ER-

$106 \mathrm{CT}$ is also a non-destructive method and can give sugar content distribution including 
107 the center part of a fruit where IR light can't reach. Our goal is to estimate the sugar

108 content of fruit samples with accuracy of $1.0 \%$.

109 In this report, we first describe a method to estimate the sugar content of

110 fructose solutions in an acrylic (PMMA) container using standard phantoms. After

111 establishing this method, it is used to estimate the sugar content of kiwifruit samples.

\section{2. Materials and Methods}

\subsection{Experimental setup for ER-CT}

115 The experimental setup for ER-CT is shown in Figure 1. The X-ray tube (TRIX-150S,

116 Toreck Co., Ltd., Yokohama, Japan) had a W target with a colliding angle of $22^{\circ}$. This

117 X-ray tube had a $1 \mathrm{~mm}$ thick Be filter. An additional Al filter with a thickness of $2 \mathrm{~mm}$

118 was included in the system to decrease the number of low-energy X-rays. A rotating

119 wheel consisting of a PMMA disk with a thickness of $1 \mathrm{~mm}$ was placed at the X-ray

120 tube exit to change the X-ray energy spectra. The rotating wheel was divided into four

121 regions - one uncovered region and three covered with $\mathrm{Cu}$ filters with thicknesses of 0.1 ,

1220.2 , and $0.3 \mathrm{~mm}$ - which provided four different X-ray energy spectra.

123 X-rays were measured by a flat panel detector (FPD; Remote RadEye2,

124 Teledyne Rad-icon Imaging, Santa Clara CA, USA). The FPD contained a $1024 \times 1024$

125 array of $48 \times 48 \mu \mathrm{m}$ silicon photodiode pixels. The sensitive area of the FPD was $48.2 \times$

$12648.2 \mathrm{~mm}$. X-rays were absorbed by a $\mathrm{Gd}_{2} \mathrm{O}_{2} \mathrm{~S}$ (GOS) scintillator plate with an effective

127 thickness of $50 \mu \mathrm{m}$. Scintillation photons were detected by the photodiode pixels.

128 The FPD and a region on the rotating wheel corresponded to a segmented

129 detector of the stacked-type transXend detector; thus, the two-dimensional transXend

130 detector in this experimental setup had four segmented detectors. Each segmented 
131 detector is hereafter denoted as a channel: $\mathrm{Cu}$ filters with thicknesses of $0,0.1,0.2$, and

$1320.3 \mathrm{~mm}$ correspond to channel $1,2,3$, and 4, respectively. X-ray spectra for channel 1

133 and 4 calculated using the formula of Birch et al. [17] are shown in Figure 2.

134 To suppress scattered X-rays, a 1-mm-thick W plate with a horizontal opening

135 with a width of $4 \mathrm{~mm}$ was placed between the X-ray tube and a phantom. The phantom

136 was placed on a precision rotating stage (SGSP-60YAW, Sigma Koki, Tokyo, Japan)

137 for CT measurements from 36 angles. The operating conditions of the X-ray tube were $138100 \mathrm{kV}, 2.4 \mathrm{~mA}$, and $0.5 \mathrm{~s}$ for each CT measurement angle.

\subsection{Standard phantoms for sugar content measurements}

140 As preparation for the sugar content estimation of fruit samples by ER-CT, fructose

141 solutions were used as known liquid. Fructose solutions with various sugar contents

142 were added to a hole with a 15-mm diameter in a PMMA cylinder with a diameter of 30

$143 \mathrm{~mm}$ to form standard phantoms.

144 The chemical formula of fructose is $\mathrm{C}_{6} \mathrm{H}_{12} \mathrm{O}_{6}$ and its effective atomic number is

1456.95 according to an empirical formula reported by Mayneord [18]. We made fructose

146 solutions with mass percent concentrations of $5 \%, 10 \%, 15 \%$, and $30 \%$. The sugar

147 contents of these solutions were measured by a sugar refractometer (APAL-J, Atago,

148 Tokyo, Japan). The measured sugar contents for the $5 \%, 10 \%, 15 \%$, and $30 \%$ solutions

149 were $4.9,9.8,15.0$, and $29.7 \pm 0.2^{\circ} \mathrm{Bx}$, respectively.

\section{$150 \quad 2.3$ Analysis}

\section{$151 \quad 2.3 .1$ Response functions}

152 To estimate X-ray energy spectra after passing through a phantom with various paths,

153 response functions for each channel are necessary. Response functions were estimated

154 using the measured electric currents induced by X-rays that passed through reference 
155 PMMA slabs with thicknesses of 10, 20, 30, and $40 \mathrm{~mm}$ which are denoted as reference material No. 1, 2, 3, and 4, respectively. The electric current measured after passing through the $j$-th reference material in

158 the $i$-th channel is written as

$I_{i, j}=a \int Y(E) R_{i}(E) \exp \left\{-\mu_{P}(E) t_{j}\right\} d E+b$.

160 Here, $Y(E)$ is the number of X-ray photons with energy $E$ emitted by the X-ray tube,

$161 R_{i}(E)$ is the response function of $i$-th channel, $\mu_{P}(E)$ is the linear attenuation

162 coefficient of PMMA, and $t_{j}$ is the thickness of PMMA as the $j$-th reference material.

163 Also, $a$ and $b$ are the correction factors for gain and offset, respectively. The X-ray

164 spectrum $Y(E)$ is calculated as,

$$
Y(E)=Y_{0}(E) \exp \left\{-\mu_{A l}(E) t_{A l}-\mu_{W}(E) t_{W}\right\}
$$

Here, $Y_{0}(E)$ is the $\mathrm{X}$-ray energy spectrum calculated using the formula developed by

167 Birch et al., $\mu_{A l}(E)$ and $\mu_{W}(E)$ are the linear attenuation coefficients of $\mathrm{Al}$ and $\mathrm{W}$,

168 respectively, and $t_{A l}$ and $t_{W}$ are the thicknesses of $\mathrm{Al}$ and $\mathrm{W}$, respectively, used to correct $Y_{0}(E)$ to reproduce measured electric current. The $\mathrm{W}$ thickness correction is for taking into account the Heel effect, which is influenced by the X-ray path length inside the W target. [19] The response function $R_{i}(E)$ can be written as,

$$
R_{i}(E)=R_{0}(E) \exp \left\{-\mu_{C u}(E) t_{i} c_{i}\right\},
$$

where $R_{0}(E)$ is the energy deposited in the GOS scintillator by an X-ray photon with energy $E$ calculated by the GEANT4 Monte Carlo code [20] and $\mu_{C u}(E), t_{i}$, and $c_{i}$ are the linear attenuation coefficient of $\mathrm{Cu}$, the thickness of the $\mathrm{Cu}$ filter, and the correction

176 factor for the thickness of the $i$-th channel $\mathrm{Cu}$ filter $(i=1,4)$, respectively.

178 following relationship,

$179 S=\sum_{i, j}\left(I_{i, j}^{\text {meas. }}-I_{i, j}\right)^{2}$. 
180 Here, $I_{i, j}^{\text {meas. }}$ is the measured electric current for X-rays passing through the $j$-th

181 reference material in the $i$-th channel, which was averaged over horizontal pixels of the

182 FPD from 1 to 1024 and also averaged vertically from the 486th to the 500th lines of

183 pixels in the FPD to avoid the possible fluctuation of pixel values. Schematic drawing

184 of this process is shown in Figure 3. Obtained response functions are shown in Figure 4.

\subsubsection{Material decomposition method}

187 In this study, we used a material decomposition method to estimate the X-ray energy

188 distribution after passing through a phantom. As shown in Figure 1, the measured

189 electric current induced by X-rays in the $k$-th horizontal pixel of the FPD at the $l$-th

190 rotating angle of the phantom in the $i$-th channel is written as,

$191 \quad I_{i, k, l}=a \int Y(E) R_{i}(E) \exp \left\{-\mu_{k, l}(E) d_{k, l}\right\} d E+b$.

192 The thicknesses of PMMA and the fructose solution change according to the measurement position $(k, l)$. We measured four electric currents for each measurement

194 position $(k, l)$. However, we have 161 unknown $\mu_{k, l}(E) d_{k, l}$, because we estimated the

195 X-ray energy spectrum every $0.5 \mathrm{keV}$ from 20 to $100 \mathrm{keV}$. It is therefore very difficult

196 to determine $\mu_{k, l}(E) d_{k, l}$ from only four measurement values at each measurement

197 position $(k, l)$ by unfolding analysis.

198 To overcome this difficulty, we reproduced $\mu_{k, l}(E) d_{k, l}$ using the linear 199 attenuation coefficients of hydrogen $\mu_{H}(E)$ and oxygen $\mu_{O}(E): Z_{\text {eff }}$ of fructose solutions 200 ranges from 6.95 (fructose) to 7.42 (water) and that of PMMA is 6.47. The atomic 201 numbers of hydrogen and oxygen are 1 and 8, respectively, and $Z_{\text {eff }}$ of the fructose 202 solutions and PMMA are between these values. The linear attenuation coefficient of a

203 material with $Z_{\text {eff }}$ between 1 and 8 can be reproduced by the linear combination of 
$204 \mu_{H}(E)$ and $\mu_{O}(E)$. For example, the linear attenuation coefficient of carbon is

205 reproduced as,

$206 \mu_{C}(E)=6.4 \times 10^{3} \mu_{H}(E)+4.8 \times 10^{2} \mu_{O}(E)$

207 as shown in Figure 5.

208 Equation (5) can be rewritten using $\mu_{H}(E)$ and $\mu_{O}(E)$ as,

$I_{i, k, l}=a \int Y(E) R_{i}(E) \exp \left\{-\mu_{H}(E) d_{k, l}^{H}-\mu_{O}(E) d_{k, l}^{O}\right\} d E+b$.

210 Here, $d_{k, l}^{H}$ and $d_{k, l}^{O}$ are the thicknesses of hydrogen and oxygen, respectively, to

211 reproduce the X-ray attenuation at measurement position $(k, l)$. To estimate unknown

212 values $d_{k, l}^{H}$ and $d_{k, l}^{O}$, we prepared look-up tables (LUTs) for the thicknesses of hydrogen

213 and oxygen, $d^{H}$ and $d^{O}$, respectively, for each channel. We estimated $d_{k, l}^{H}$ and $d_{k, l}^{O}$ by

214 finding $d^{H}$ and $d^{O}$ that minimized the following equation,

$215 S_{k, l}=\sum_{i=1}^{4}\left\{I_{k, l}^{\text {meas., } i}-I^{i}\left(d^{H}, d^{O}\right)\right\}^{2}$,

216 with changing $d^{H}$ and $d^{O}$ from zero value to their maximums, $d^{H, \max }$ and $d^{O, \max }$. The

217 electric current $I_{k, l}^{\text {meas.i }}$ was obtained by averaging vertically from the 486th to the 500th

218 lines of the pixels in the FPD. $I^{i}\left(d^{H}, d^{O}\right)$ is the electric current value in the LUT

219 prepared for the $i$-th channel as functions of $d^{H}$ and $d^{O}$. The schematic drawing of

220 finding $d^{H}$ and $d^{O}$ is shown in Figure 6.

221

\subsubsection{Water phantom correction}

223 Sugar content was estimated by comparing measured and theoretical linear attenuation coefficients. Measured linear attenuation coefficients were obtained from the ER-CT

225 images as CT values as a function of X-ray energy. To have correct CT values, the 226 transmission measurement of X-rays through water should be the same as that 
227 calculated using the Lambert-Beer law [21]. Figure 7 shows the theoretical and 228 experimental results of X-ray transmission through a water phantom (i.e., zero sugar content). Although the results in Figure 7 look fairly similar, they differ slightly because of X-ray scattering and other effects. The ratio of theoretical to experimental values is

231 presented in Figure 8. This ratio was applied to all measured results as the water 232 phantom correction factor.

\subsection{Sugar contents of standard phantoms}

235 A reconstructed CT image using the number of X-ray photons with an energy of $70 \mathrm{keV}$

236 for a $10 \%$ fructose solution is shown in Figure 9. The CT value of this image shows the

237 linear attenuation coefficient for X-rays with an energy of $70 \mathrm{keV}$. By assigning a $23850 \times 50$ pixel region in the fructose solution as the region of interest $(\mathrm{ROI})$, the linear 239 attenuation coefficient as a function of X-ray energy was obtained as the averaged CT 240 value in the ROI. The linear attenuation coefficients for $5 \%, 15 \%$, and $30 \%$ fructose

241 solutions as a function of X-ray energy are displayed in Figure 10. Error bars given by 242 the standard deviations of the CT values in ROIs are only shown for the linear attenuation coefficient of the $30 \%$ fructose solution because the error bars for the linear attenuation coefficients were similar for all solutions with different sugar contents.

246 the linear attenuation coefficients as a function of X-ray energy, $\mu_{r e f .}\left(f_{i}, E_{j}\right)$, for

247 fructose solutions with the concentration of $f_{i}$ with $0.1 \%$ intervals from 0 to $30 \%$ by 248 calculations using data from NIST [1]. The sugar content of fructose solutions was

249 estimated by finding $\mu_{r e f .}\left(f_{i}, E_{j}\right)$ which gives the minimum value of $S_{s}\left(f_{i}\right)$ in the 250 following equation, 


$$
S_{S}\left(f_{i}\right)=\sum_{j=1}^{101}\left\{\mu_{\text {meas. }}\left(E_{j}\right)-\mu_{\text {ref. }}\left(f_{i}, E_{j}\right)\right\}^{2}
$$

252 Here, $\mu_{\text {meas. }}\left(E_{j}\right)$ is the measured linear attenuation coefficient at energy $E_{j}$. We used 253 linear attenuation coefficients for X-ray photon energies from 40 to $90 \mathrm{keV}$ for the 254 sugar content estimation because of the small numbers of X-ray photons with energies 255 below $40 \mathrm{keV}$ and above $90 \mathrm{keV}$. Estimated sugar contents are shown in Figure 11. 256 The calibration line obtained using least squares fitting is drawn as a solid line. The 257 dashed lines show the limitation of $\pm 1 \%$ from the calibration line.

Flow chart of sugar content estimation method is shown in Figure 12.

\subsection{Sugar content measurements of kiwifruit}

260 We applied the sugar content estimation method described above to two kiwifruit

261 samples. Because the dimensions of the sensitive area of our FPD was $48.2 \times 48.2 \mathrm{~mm}$

262 and the diameters of commercially available kiwifruits are greater than $50 \mathrm{~mm}$, we cut

263 kiwifruit samples out with diameters of less than $24 \mathrm{~mm}$ and put them in PMMA

264 casings with inner and outer diameters of 24 and $30 \mathrm{~mm}$, respectively: in the CT

265 measurement, the attenuation of X-rays was measured as the ratio of the electric current

266 induced by X-rays after transmitting through a sample to that of X-rays passing through

267 air. Photographs of the prepared kiwifruit samples are depicted in Figure 13. To

268 estimate the sugar contents of both the green and white parts of the kiwifruit samples, we cut samples off the axis of kiwifruits.

The ER-CT images reconstructed from the number of X-rays with an energy of

$27170 \mathrm{keV}$ are shown in Figure 14. We defined two ROIs for each CT image, which 272 corresponded to the green $\left(\mathrm{ROI}_{\mathrm{G}}\right)$ and white $\left(\mathrm{ROI}_{\mathrm{W}}\right)$ parts of the kiwifruit samples.

273 Each ROI had 50×50 pixels. Obtained linear attenuation coefficients for the kiwifruit 
samples are shown in Figure 15. Error bars are shown only for the linear attenuation coefficients of green parts because those for the white parts had nearly the same lengths. Using Equation (9), the sugar contents of the kiwifruit samples were estimated.

The estimated sugar contents of the green part of the kiwifruit samples are presented in Figure 11, in which the $x$-values show those measured by the refractometer for juice squeezed from the green part of each sample.

\section{Results and Discussion}

282 The transmission measured for the water phantom looked to agree well with the

283 theoretical one in Figure 7; however, the slight difference between them is shown in

284 Figure 8. The cause of this difference is not obvious, but we think that scattered X-rays 285 may be the main reason. Large deviations can be seen for the pixel number 115 and 903. This is because the borders of these pixels did not match with that between PMMA and water. We consider that this difference is a geometric effect. Therefore, a geometrical correction was carried out by multiplying all the measured transmission data by this factor.

Ring artifacts are seen in Figure 9. We think the artifacts were brought by the small number of X-ray photons and also the small number of projection angles. Similar artifacts are seen in Figure 14.

Figure 10 displays the error bars at every $10 \mathrm{keV}$ for the linear attenuation coefficient of $30 \%$ fructose solution. The error bar at $40 \mathrm{keV}$ was the smallest. We

295 think the smallest error bar was observed at $40 \mathrm{keV}$ because of the large number of Xray photons of this energy in channel 1 (i.e., without a $\mathrm{Cu}$ filter), as shown in Figure 2.

297 The error bars are larger than those of Figure 5 of Ref. [10]. In Ref. [10], the X-rays 298 were collimated by two $\mathrm{W}$ collimators with a diameter of $1 \mathrm{~mm}$. In this case, the X-ray 
299 path was well defined so there was almost no effect from scattered X-rays. In the

300 estimation of fruit sugar content or the ER-CT imaging of a human body, however, we

301 have to use a two-dimensional transXend detector, so the influence of X-ray scattering

302 cannot be avoided. The development of an algorithm that omits scattered X-rays will be

303 the focus of our future study.

304 It may be possible to estimate the error of sugar content values by fitting linear

305 attenuation coefficients taking the upper and lower values of error bars. However, this

306 estimation does not have much meaning. Because the center value of linear attenuation

307 coefficients changes according to the sugar content of the samples, we believe that our

308 ER-CT measurements were performed properly and the error was systematic: small

309 number of X-ray photons.

310 The sugar contents of the green parts of kiwifruit samples showed good

311 agreement with those measured by the refractometer within the error of $1 \%$ from the

312 calibration line, which was the purpose of this study. We could not extract juice from

313 the white parts of the kiwifruit samples but had white suspension, so we could not

314 measure their sugar content with the refractometer. The sugar content estimated by ER-

315 CT was $1.1^{\circ} \mathrm{Bx}$ for the white part of kiwifruit sample (a). The linear attenuation

316 coefficient of the white part of kiwifruit sample (b) was smaller than that of water so we

317 could not estimate its sugar content. The estimated $Z_{\text {eff }}$ of the white part of kiwifruit

318 sample (b) was 7.72, which was greater than that of water (7.42).

319 Using ER-CT, we can obtain the distribution of sugar content in a fruit sample.

320 The green and white parts of a kiwifruit showed different sugar contents. In IR

321 spectroscopy, sugar content can be measured only for regions close to the fruit surface.

322 Therefore, the sugar content measurement by ER-CT has an advantage over that

323 determined by IR spectroscopy regarding this point. The developed sugar content 
324 estimation method using ER-CT can be used for other non-destructive food inspections

325 such as fat content measurement. To extend the application of the two-dimensional 326 transXend detector, a method to suppress the effect of X-ray scattering should be

327 developed in the future.

328

329 Acknowledgment

330 We thank Dr. Y. Yamashita, Osaka Prefecture University College of Technology, for

331 fruitful discussion. We acknowledge The Kansai Atomic Conference for financial 332 support.

334 References

335 [1] Hubbell JH, Seltzer SM. Tables of X-ray mass attenuation coefficients and mass 336 energy-absorption coefficients from $1 \mathrm{keV}$ to $20 \mathrm{MeV}$ for elements $Z=1-92$ and 48

337 additional substances of dosimetric interest. The Physical Measurement Laboratory, The

338 National Institute of Standard and Technology; 2010. Available from:

339 http://www.nist.gov/pml/data/xraycoef/index.cfm

340 [2] Brooks RA, Di Chiro G. Beam hardening in X ray reconstructive tomography. Phys

341 Med Biol. 1976;21:390-398.

342 [3]Poumorteza A, Syons R, Sandfort V., et al. Abdominal imaging with contrast-

343 enhanced photon-counting CT:First human experience. Radiol. 2016;279:239-245.

344 [4] Bazalova M, Carrier J-F, Beaulier L, et al. Tissue segmentation in Monte Carlo

345 treatment planning: a simulation study using dual-energy CT images. Radiother Oncol. $346 \quad 2008 ; 86: 93-98$.

347 [5] van Elmpt W, Landry G, Das M, et al. Dual energy CT in radiotherapy: current 348 applications and future outlook. Radiother Oncol. 2016;119:137-144. 
349 [6] Le HQ, Ducote JL, Molloi S. Radiation dose reduction using a CdZnTe-based

350 computed tomography system: comparison to flat-panel detectors. Med Phys. 2010; 37:

$351 \quad 1225-1236$.

352 [7] de Vries A, Roessl E, Kneepkens E, et al. Quantitative spectral K-edge imaging in

353 preclinical photoncounting X-ray computed tomography. Invest Radiol. 2015;50:297-

354304.

355 [8] Atak H, Shikhaliev PM. Dual energy CT with photon counting and dual source systems: comparative evaluation. Phys Med Biol. 2015;60:8949-8975.

357 [9] Kanno I, Imamura R, Mikami K, et al. A current mode detector for unfolding X-ray 358 energy distribution. J Nucl Sci Technol. 2008;45:1165-1170.

359 [10] Hamaguchi T, Kanno I. Effective atomic number estimation by energy-resolved X-

360 ray computed tomography with a current-mode detector system. Jpn J Appl Phys. 2019;

361 58: 071001-6.

362 [11] Kanno I, Yamashita Y, Kanai E, Ogawa T, Shinsho K. Two-dimensional

363 "transXend" detector for third-generation energy-resolved computed tomography. J

364 Nucl Sci Tehcnol. 2016;53:258-262.

365 [12] Constantinou C, Attix FH, Paliwal B. A solid water phantom material for 366 radiotherapy x-ray and gamma ray beam calibrations. Med Phys. 1982;9:436-441.

367 [13] Iramina H, Nakamura M, Mizowaki T, Kanno I. Effective atomic number 368 measurement with energy-resolved computed tomography using two-dimensional 369 “transXend” detector. Int J Med Phys Clin Eng Radiat Oncol. 2018;7:61-73.

370 [14] Bazalova M, Carrier JF, Beaulieu L, Varhaegen F. Dual-energy CT-based metarial extraction for tissue segmentation in Monte Carlo dose calculations. Phys Med \& Biol. 2008;53:2439-2456. 
373 [15] Goodsitt MM, Christodoulou EG, Larson SC. Accuracies of the synthesized

374 monochromatic CT numbers and effective atomic numbers obtained with a rapid $\mathrm{kVp}$

375 switching dual energy CT scanner. Med Phys. 2011;38:2222-2232.

376 [16] https://www.atago.net/en/pdf/tanpin-catalog/pal-hikari5 10 12_en.pdf.

377 [17] Birch R, Marshall M. Computation of bremsstrahlung X-ray spectra and

378 comparison with spectra measured with a Ge(Li) detector. Phys Med Biol. 1979;24:505-

379517.

380 [18] Mayneord W V. The significance of the Röntgen. Acta Int. Union against Cancer $381 \quad 1937 ; 2: 271-282$.

382 [19] Buzug T M. Computed Tomography, Heidelberg:Springer; 2010.

383 [20] Agostinelli S, Allison J, Amako K, et al. GEANT4 -a simulation toolkit. Nucl

384 Instrum Methods Phys Res. 2003;A506:250-303.

385 [21] Hsieh J. Computed tomography. 2nd ed. Washington:SPIE Press; 2009.

386

387

388

389

390

391

392

393

394

395

396

397 
Figure captions

399

400 Figure 1. Experimental setup for sugar content measurements using a two-dimensional

401 transXend detector with four channels.

402

403 Figure 2. Calculated X-ray energy spectra for channel 1 and 4.

404

405 Figure 3. Schematic drawing of determining correction factors. Correction factors, $a, b, t_{A l}, t_{W}, c_{1}, c_{2}, c_{3}, c_{4}$ are determined as the ones which result in the minimum $S$ while changing the values of correction factors.

408

409

Figure 4. Response functions of the transXend detector for each channel. The valleys at $50 \mathrm{keV}$ correspond to the K-edge of Gd.

411

412 Figure 5. The linear attenuation coefficient of carbon (solid line) reproduced by a linear

413 combination of those of hydrogen (dashed line) and oxygen (dotted line) given by NIST.

414 Solid circles are the theoretical linear attenuation coefficient of carbon taken from NIST 415 data.

416

417 Figure 6. Schematic drawing of material decomposition, i.e., finding the thicknesses of

418 hydrogen and oxygen to reproduce the X-ray attenuation by a subject. $(m, n)$ in a small

419 square mean the estimated electric current with taking into account the X-ray 420 attenuation with the thicknesses of hydrogen, $d^{H, m}$ and oxygen, $d^{O, n}$ in $i$-th channel.

$421 d^{H, \max }$ and $d^{O, \max }$ are the maximum values of hydrogen and oxygen thicknesses 422 prepared for the look up table. 
424 Figure 7. Measured and theoretically obtained electric current induced by X-rays after transmitting a water phantom.

426

427 Figure 8 . Correction factor for the water phantom obtained as the ratio of theoretical 428 value to measured value in Figure 7.

429

430 Figure 9. ER-CT image reconstructed for the number of X-rays with an energy of 70 $431 \mathrm{keV}$ for $10 \%$ fructose solution in an acrylic cylinder.

432

433 Figure 10. Measured linear attenuation coefficients for 5\%, 15\%, and 30\% fructose 434 solutions as the averaged values of $50 \times 50$ pixel ROI in ER-CT images obtained at 435 intervals of $0.5 \mathrm{keV}$ from 20 to $90 \mathrm{keV}$. Error bars are shown only for $30 \%$ fructose solution every $10 \mathrm{keV}$.

437

438 Figure 11. Estimated sugar contents of 5\%,10\%, 15\%, and 30\% fructose solutions 439 (open circles) and the green parts of two kiwifruit samples (solid circles). The solid line

440 is the calibration line given by the least squares fitting. Dashed lines show the $\pm 1 \%$ 441 ranges from the calibration line.

442

443 Figure 12. Flow chart of sugar content estimation. "LUT" means look up table.

445 Figure 13. Photographs of kiwifruit samples in acrylic casings. 
447 Figure 14. ER-CT images of kiwifruit samples reconstructed from the number of X-rays

448 with an energy of $70 \mathrm{keV}$. ROIs for green and white parts are shown.

449

450 Figure 15. Obtained linear attenuation coefficients for green (solid lines) and white 451 (dashed lines) parts of kiwifruit samples. Error bars are only shown for the linear 452 attenuation coefficients for the green part every $10 \mathrm{keV}$. The error bars for the white 453 part had similar values.

454

455

456

457

458

459

460

461

462

463

464

465

466

467 

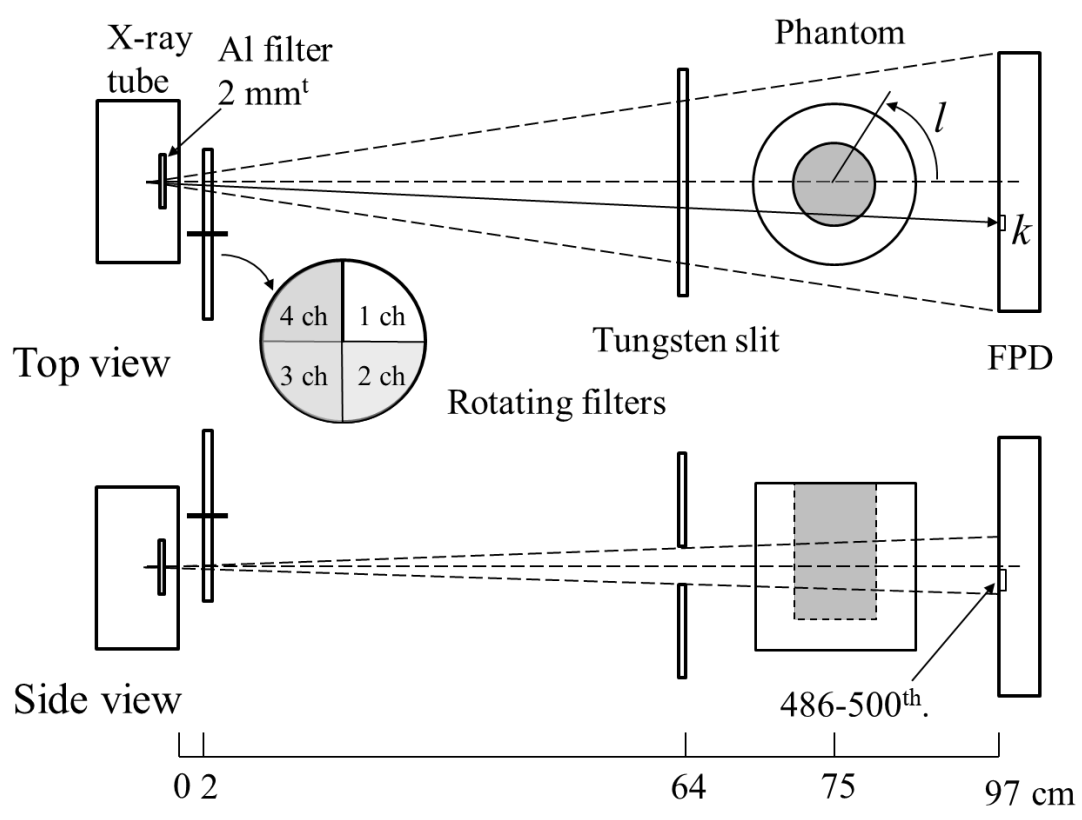

469

470

$471 \quad$ Figure 1

472

473

474

475

476

477

478

479

480

481 
482

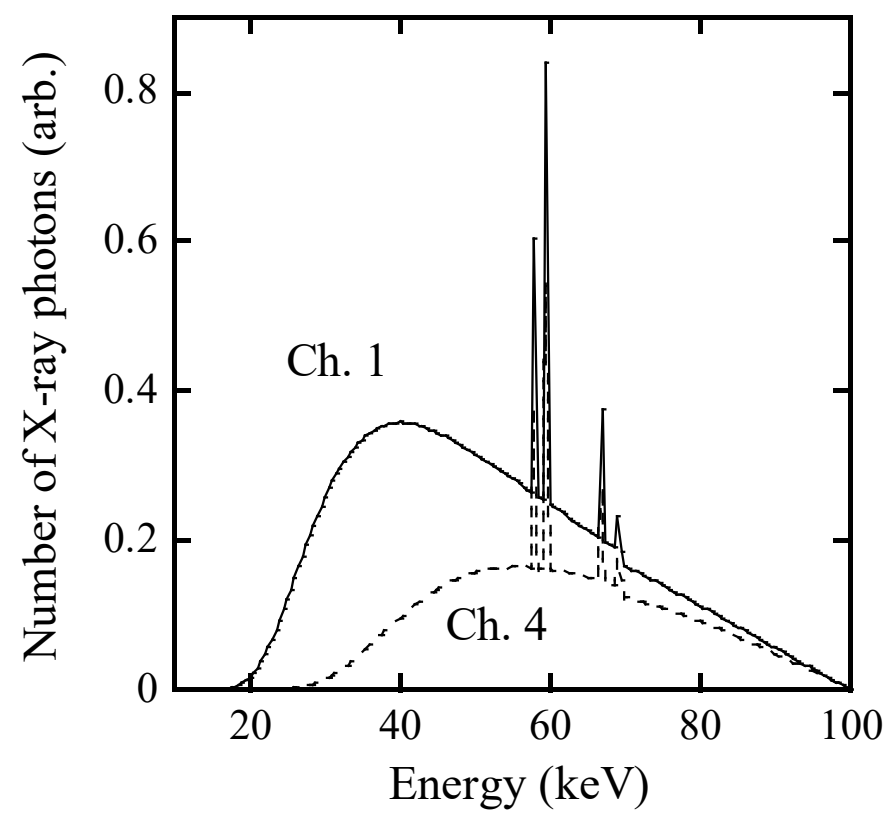

483

484 Figure 2

485

486

487

488

489

490

491

492 
493

\begin{tabular}{|c|c|c|c|c|}
\hline 4 & $I_{1,4}$ & $I_{2,4}$ & $I_{3,4}$ & $I_{4,4}$ \\
\hline 3 & $I_{1,3}$ & $I_{2,3}$ & $I_{3,3}$ & $I_{4,3}$ \\
\hline 2 & $I_{1,2}$ & $I_{2,2}$ & $I_{3,2}$ & $I_{4,2}$ \\
\hline 1 & $I_{1,1}$ & $I_{2,1}$ & $I_{3,1}$ & $I_{4,1}$ \\
\hline$j$ & 1 & 2 & 3 & 4 \\
$i$ & & & & \\
\hline
\end{tabular}

$S=\sum_{i, j}\left(I_{i, j} \text { meas. }-I_{i, j}\right)^{2}$

Minimizing $S$

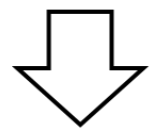

$a, b, t_{\mathrm{Al}}, t_{\mathrm{W}}, c_{1}, c_{2}, c_{3}, c_{4}$

494

$495 \quad$ Figure 3

496

497

498

499

500

501

502

503

504

505

506

507

508

509

510

511 


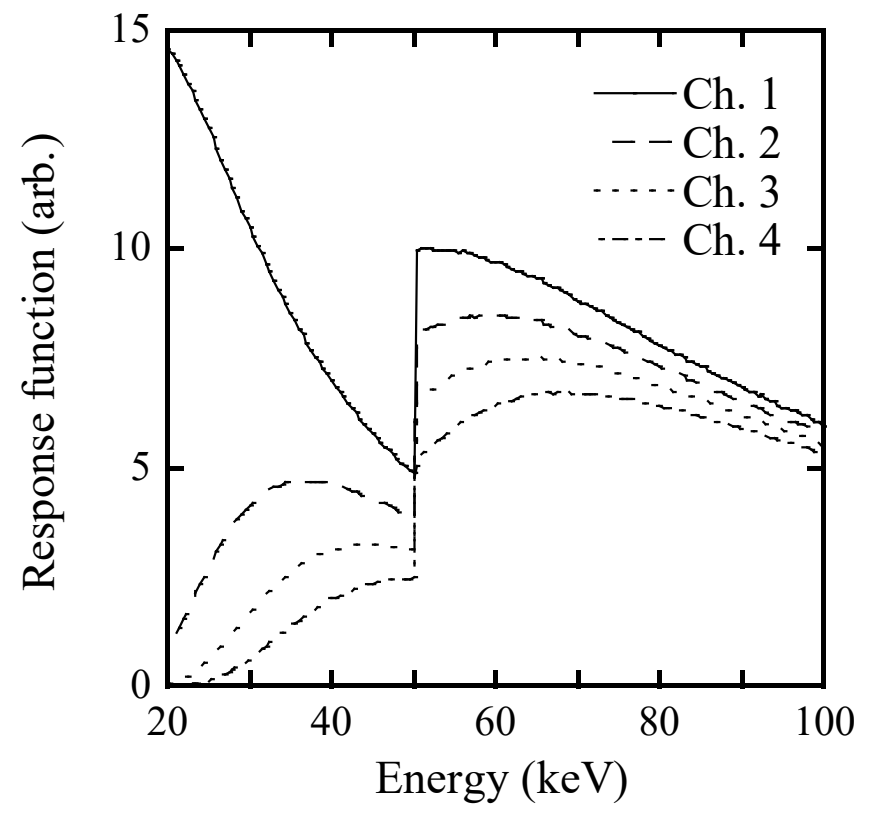

513

514

515

$516 \quad$ Figure 4

517

518

519

520

521

522

523

524

525

526

527

528 


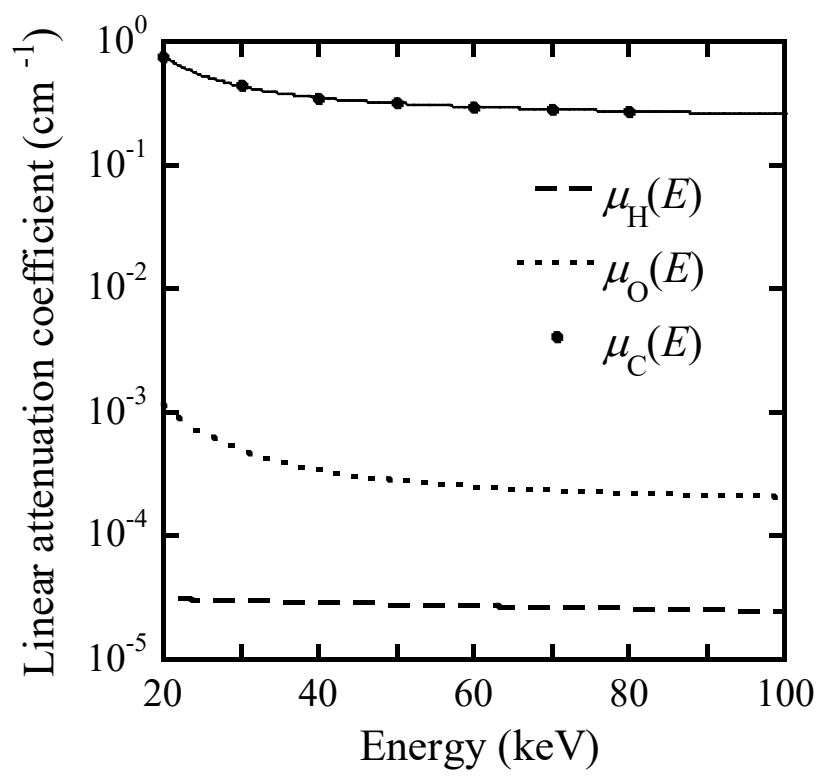

530

531

$532 \quad$ Figure 5

533

534

535

536

537

538

539

540

541

542

543

544

545 


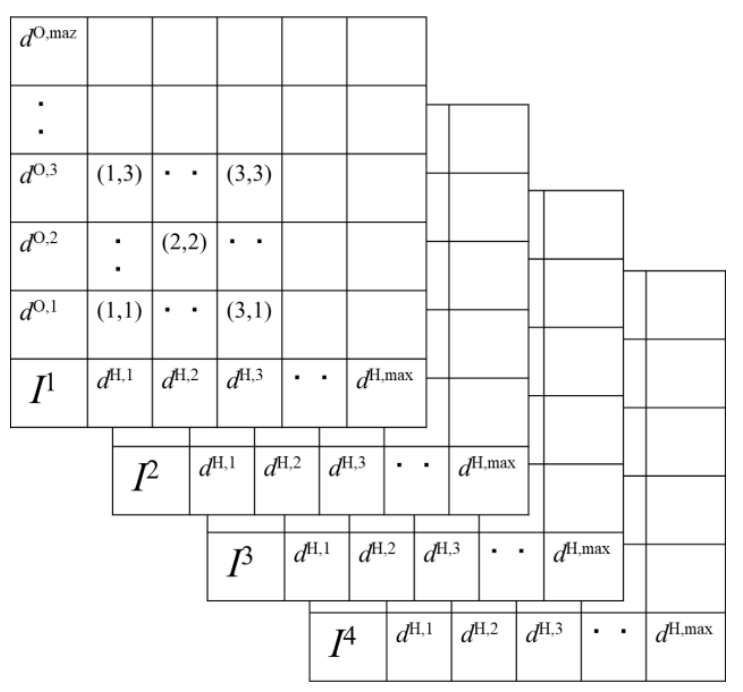

$$
S_{k, l}=\sum_{i=1}^{4}\left\{I_{k, l}^{\text {meas }, i}-I^{i}\left(d^{H}, d^{O}\right)\right\}^{2}
$$

Search minimum $S_{k, l}$ in

$$
0 \leq d^{H} \leq d^{H, \max }, \quad 0 \leq d^{O} \leq d^{O, \max }
$$

548

549

550

$551 \quad$ Figure 6

552

553

554

555

556

557

558

559

560

561

562

563 
566

567

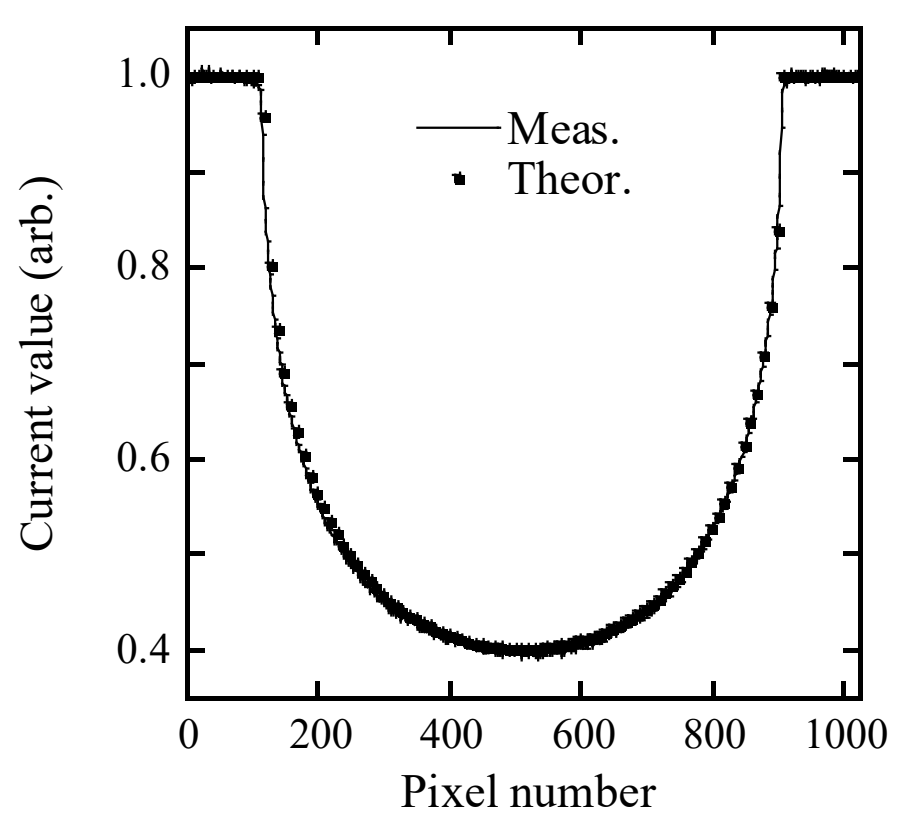

568

569

570

Figure 7

571

572

573

574

575

576

577

578

579

580

581 


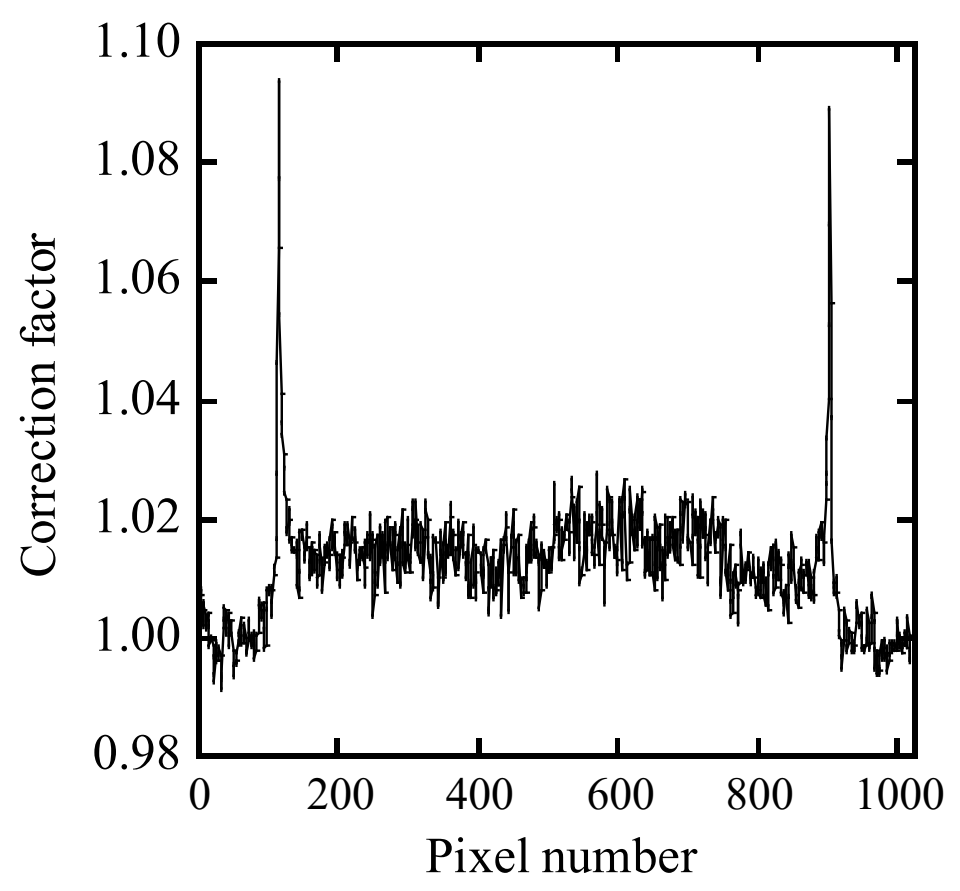

584

585

586

587

$588 \quad$ Figure 8

589

590

591

592

593

594

595

596

597 
598

599

600

601

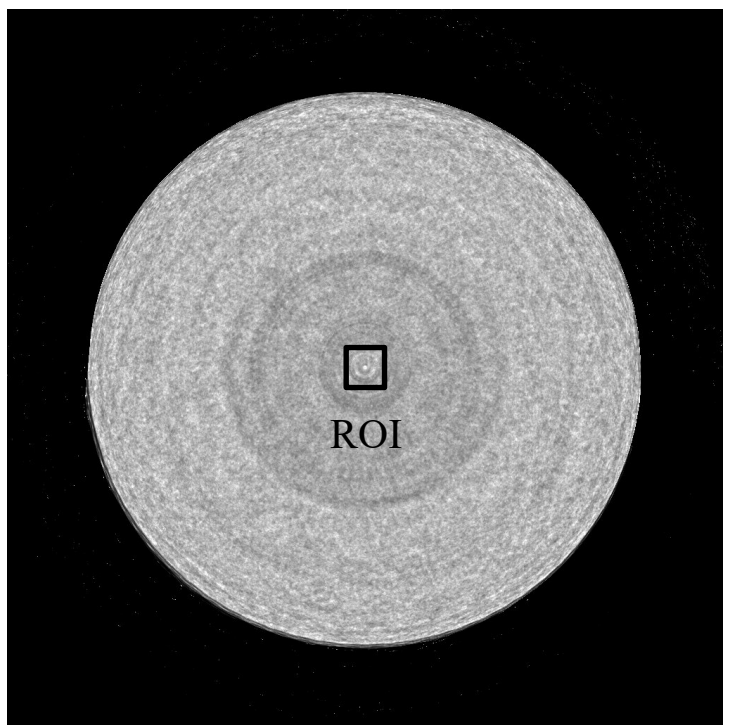

602

0.0

$0.3\left(\mathrm{~cm}^{-1}\right)$

603

604

605

606

607 Figure 9

608

609

610

611

612

613

614 


\section{5}

616

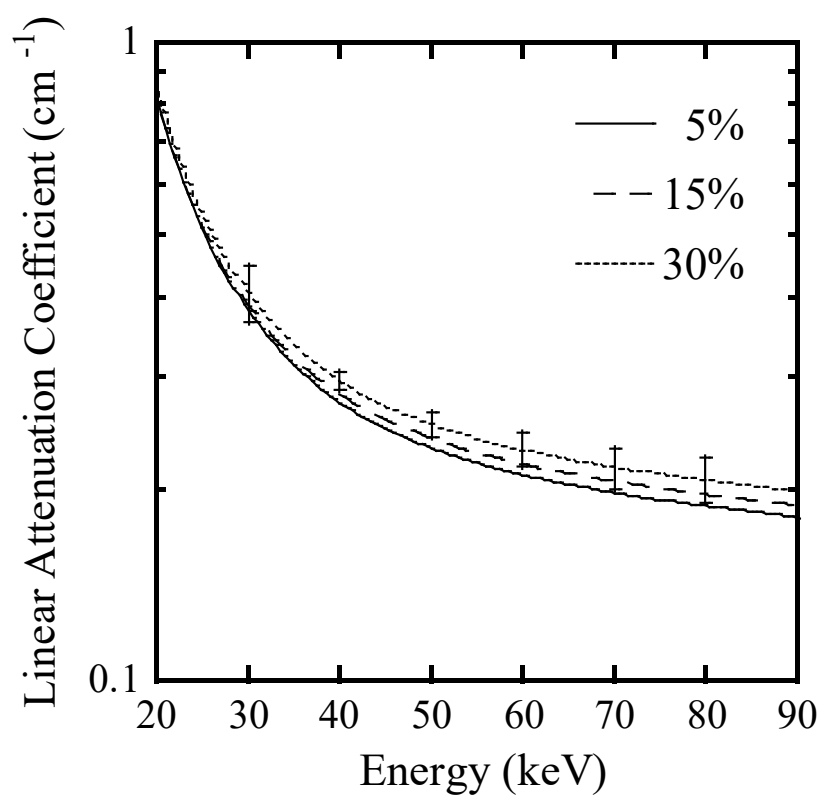

617

618

619

620

621 Figure 10

622

623

624

625

626

627

628

629

630

631 
632

633

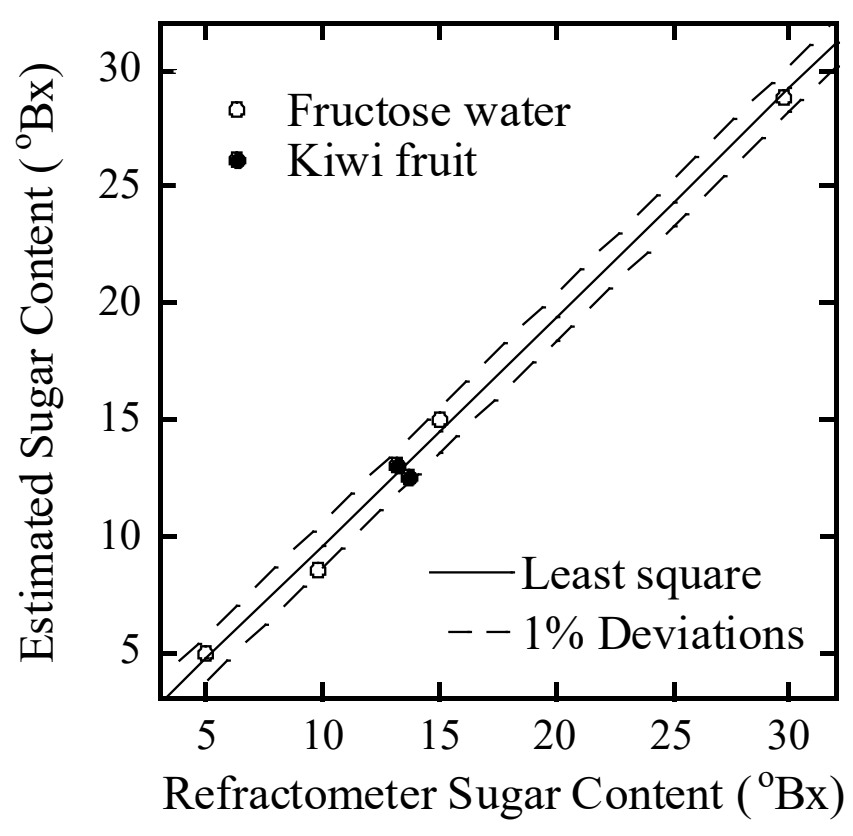

634

635

636 Figure 11

637

638

639

640

641

642

643

644

645

646

647

648 
649

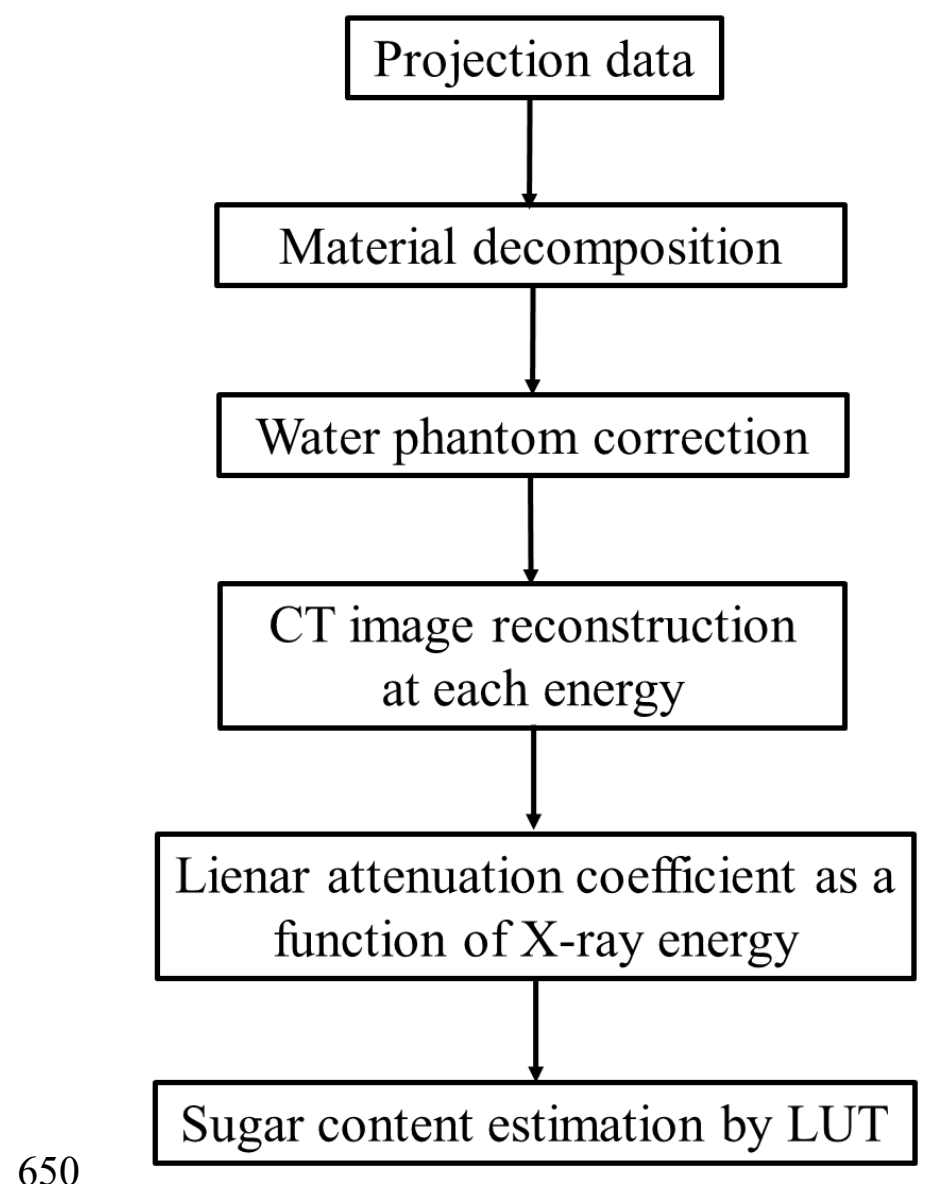

651

652 Figure 12

653

654

655

656

657 
658

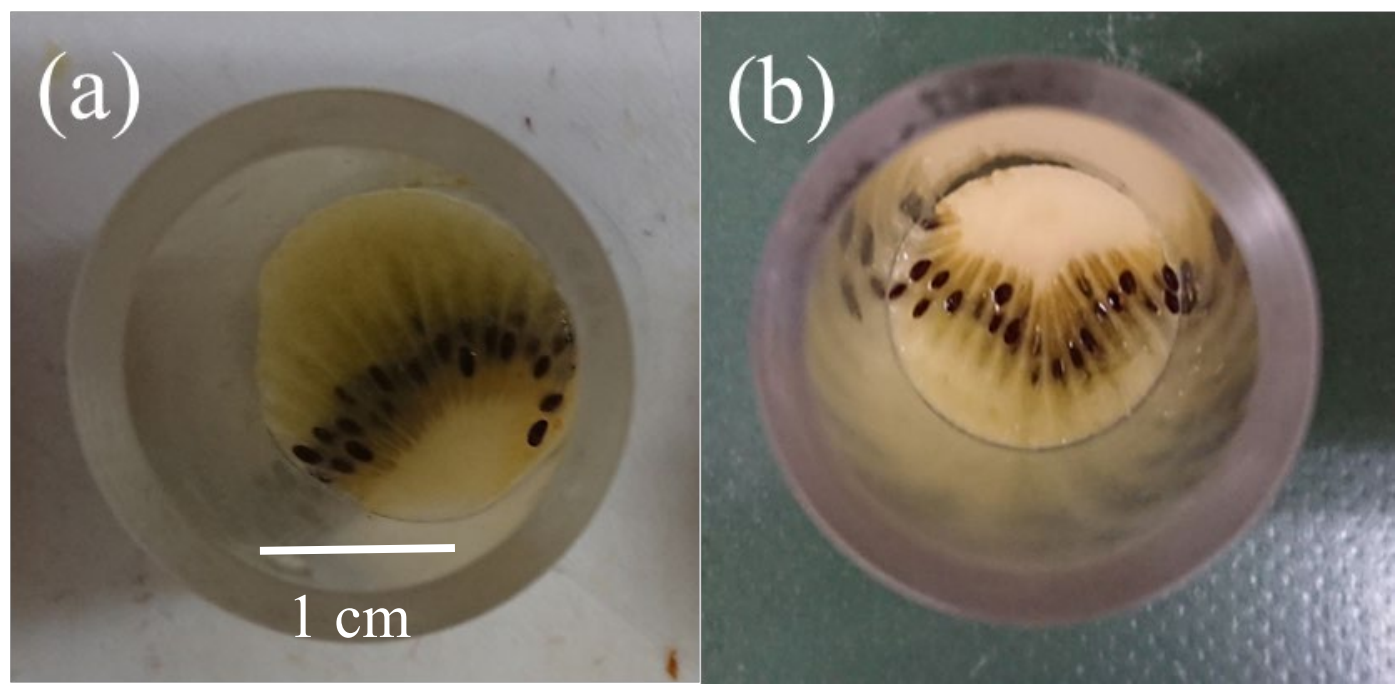

659

660 Figure 13

661

662

663

664

665

666

667

668

669

670

671

672

673

674

675

676 
677

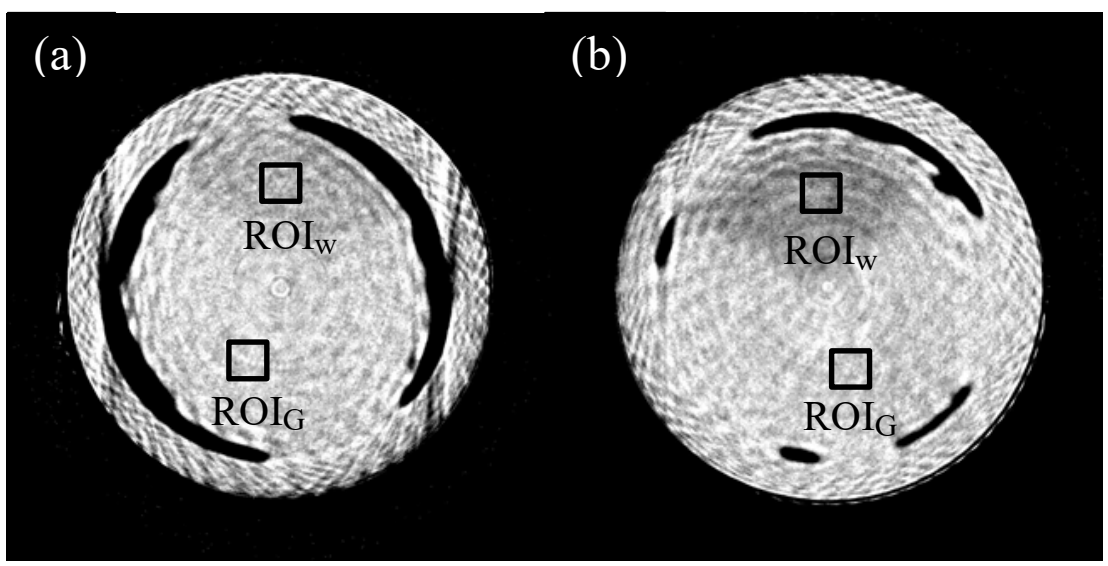

678

0.0

$0.3\left(\mathrm{~cm}^{-1}\right)$

679

$680 \quad$ Figure 14

681

682

683

684

685

686

687

688

689

690

691

692

693

694 

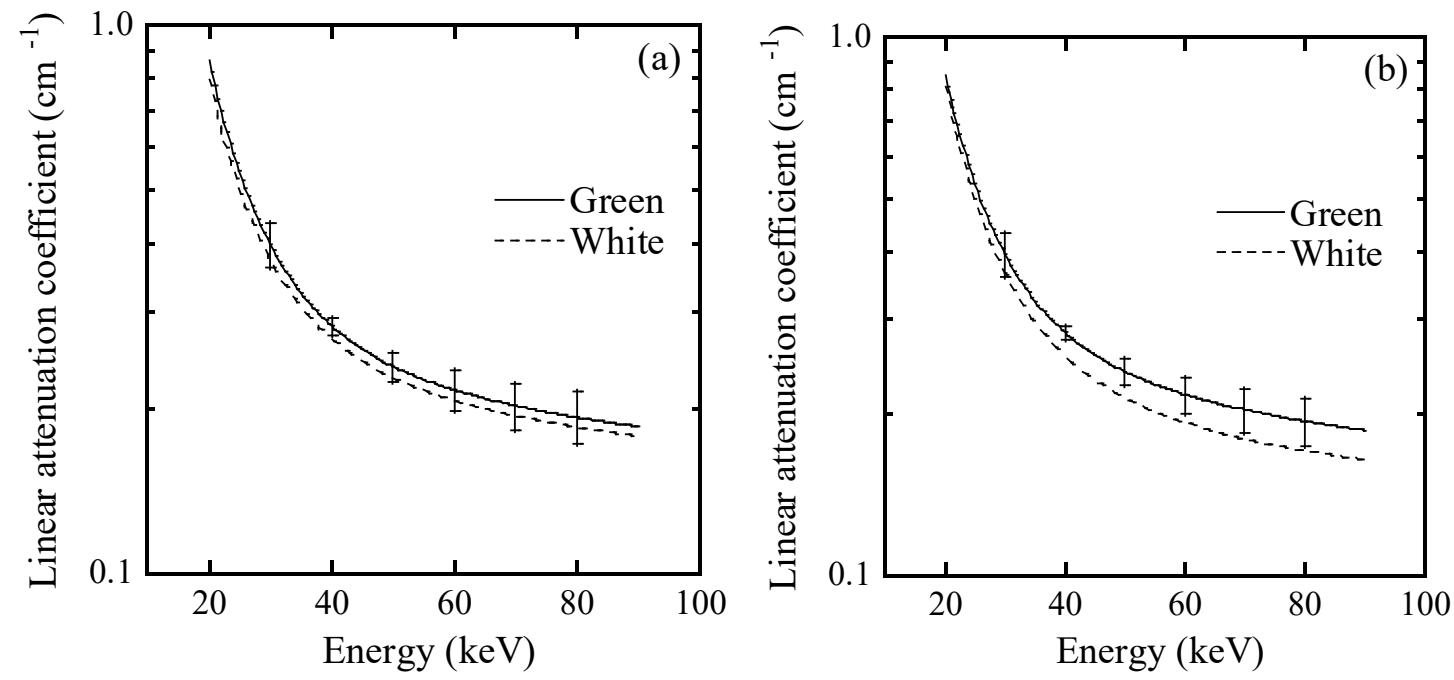

695

696

697

698 Figure 15

699

700

701

702

703

704

705

706

707

708

709

710

711 